\title{
Clinical and MRI Characteristics of Uterine Cervical Adenocarcinoma: Its Variants and Mimics
}

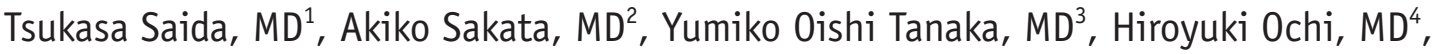 \\ Toshitaka Ishiguro, MD ${ }^{1}$, Masafumi Sakai, MD ${ }^{1}$, Hiroaki Takahashi, MD ${ }^{1}$, Toyomi Satoh, MD ${ }^{4}$, \\ Manabu Minami, $M D^{1}$ \\ Departments of ${ }^{1}$ Radiology, ${ }^{2}$ Diagnostic Pathology, and ${ }^{4}$ Obstetrics and Gynecology, Faculty of Medicine, University of Tsukuba, Tsukuba, Japan; \\ ${ }^{3}$ Department of Diagnostic Imaging, The Cancer Institute Hospital Japanese Foundation for Cancer Research, Tokyo, Japan
}

Adenocarcinoma currently accounts for $10-25 \%$ of all uterine cervical carcinomas and has a variety of histopathological subtypes. Among them, mucinous carcinoma gastric type is not associated with high-risk human papillomavirus (HPV) infection and a poor prognosis, while villoglandular carcinoma has an association with high-risk HPV infection and a good prognosis. They show relatively characteristic imaging findings which can be suggested by magnetic resonance imaging (MRI), though the former is sometimes difficult to be distinguished from lobular endocervical glandular hyperplasia. Various kinds of other tumors including squamous cell carcinoma should be also differentiated on MRI, while it is currently difficult to distinguish them on MRI, and HPV screening and pathological confirmation are usually necessary for definite diagnosis and further patient management.

Keywords: Uterus; Cervical cancer; Adenocarcinoma; MRI; Human papillomavirus

\section{INTRODUCTION}

Adenocarcinoma currently accounts for $10-25 \%$ of all uterine cervical carcinomas in developed countries (1), and its incidence just keeps increasing according to a decrease in the incidence of squamous cell carcinoma (SCC) as a result of improved screening programs (1-3). Recent studies suggest that high-risk human papillomavirus (HPV) types 16,18 , and 45 are responsible for approximately $70 \%$ SCCs and $80 \%$ adenocarcinomas (Table 1$)(1,4,5)$. The overall prognosis of adenocarcinomas is similar to or slightly poorer than that of SCC; however, the prognosis of each adenocarcinoma varies with its histopathological type (1-7).

Received July 16, 2018; accepted after revision November 5, 2018. Corresponding author: Tsukasa Saida, MD, Department of Radiology, Faculty of Medicine, University of Tsukuba, 1-1-1 Tennodai, Tsukuba 305-8575, Japan.

- Tel: (8129) 8533205 - Fax: (8129) 8533205

- E-mail: saida_sasaki_tsukasa@yahoo.co.jp

This is an Open Access article distributed under the terms of the Creative Commons Attribution Non-Commercial License (https://creativecommons.org/licenses/by-nc/4.0) which permits unrestricted non-commercial use, distribution, and reproduction in any medium, provided the original work is properly cited.
Cervical tumor is evaluated by several imaging modalities, and it has been reported that SCC tended to be hypoechoic and adenocarcinoma tended to be isoechoic on ultrasound (8), and there was no significant difference in standardized uptake value max between SCC and non-SCC on 2-[18F] fluoro-2-deoxy-D-glucose positron emission tomography (9). Computed tomography (CT) is limited by poor soft tissue contrast, and magnetic resonance imaging (MRI) is superior to CT for local evaluation (10). Some types of adenocarcinomas show characteristic imaging findings, and the histopathological type can be suggested using MRI.

The MRI findings of a variety of cervical tumors can be grossly classified into five patterns (Fig. 1): Type 1, multicystic, or solid and cystic pattern; Type 2, exophytic villous pattern; Type 3, expansile solid pattern; Type 4, invasive solid pattern; and Type 5, diffuse infiltrative pattern. Some mainly show any one of these typical imaging patterns, while others can show mixed patterns.

The purpose of this review is to discuss cervical adenocarcinomas and cervical lesions other than SCC from various aspects, including their association with high-risk HPV infection and their prognosis, and to demonstrate their representative MRI findings and patterns. 


\section{Cervical Adenocarcinoma}

Endocervical Adenocarcinoma, Usual Type (Figs. 2, 3)

This is an invasive epithelial tumor showing glandular

Table 1. Correlations of Different Uterine Cervical Tumorous Lesions with High-Risk HPV Infection

\begin{tabular}{|c|c|}
\hline Adenocarcinoma & HPV Infection \\
\hline Endocervical adenocarcinoma, usual type & + \\
\hline \multicolumn{2}{|l|}{ Mucinous carcinoma } \\
\hline GAS & - \\
\hline Intestinal type & + \\
\hline Signet-ring cell type & $+/-$ \\
\hline VGC & + \\
\hline Endometrioid carcinoma & - \\
\hline Clear cell carcinoma & - \\
\hline Serous carcinoma & - \\
\hline Mesonephric carcinoma & - \\
\hline Adenocarcinoma admixed with neuroendocrine tumor & + \\
\hline \multicolumn{2}{|l|}{ Other Tumors } \\
\hline \multicolumn{2}{|l|}{ Benign glandular tumors and tumor-like lesion } \\
\hline LEGH, nabothian cyst, endocervical polyp & - \\
\hline \multicolumn{2}{|l|}{ Other epithelial tumors } \\
\hline Adenosquamous carcinoma & + \\
\hline Neuroendocrine tumor & + \\
\hline Mesenchymal tumors & - \\
\hline \multicolumn{2}{|l|}{ Lymphoid tumors } \\
\hline Lymphoma & - \\
\hline Secondary tumors & - \\
\hline
\end{tabular}

GAS = mucinous carcinoma gastric type, HPV = human

papillomavirus, LEGH = lobular endocervical glandular hyperplasia, $\mathrm{VGC}=$ villoglandular carcinoma differentiation with relative mucin depletion. It is the most common type of cervical adenocarcinoma and accounts for approximately $90 \%$ of all adenocarcinomas. It is associated with high-risk HPV infection (Table 1) $(1,4,5)$ and, potentially, long-term oral contraceptive use. The mean age at presentation is around 50 years (1). The prognosis of the usual type adenocarcinoma is similar to that of SCC (1-7). Macroscopically, approximately 50\% tumors show an invasive growth pattern with an exophytic protrusion, and some show surface ulceration or a diffuse infiltrative pattern. Mucin-poor glands with cribriform and papillary structures are most often seen in histopathological analysis. Occasionally, cervical intraepithelial neoplasia (CIN) or adenocarcinoma in situ (AIS) coexists with this tumor, although they are frequently difficult to be detected radiologically.

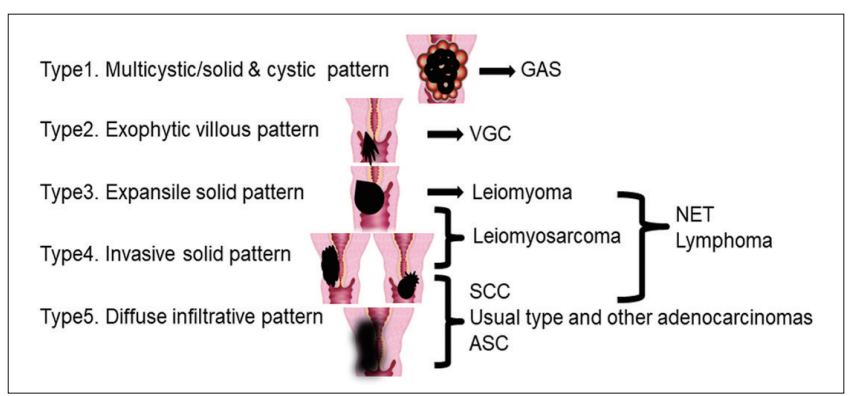

Fig. 1. MRI patterns of different cervical tumors. $A S C=$ adenosquamous carcinoma, GAS = mucinous carcinoma gastric type, MRI = magnetic resonance imaging, NET = neuroendocrine tumor, $\mathrm{SCC}$ = squamous cell carcinoma, $\mathrm{VGC}=$ villoglandular carcinoma

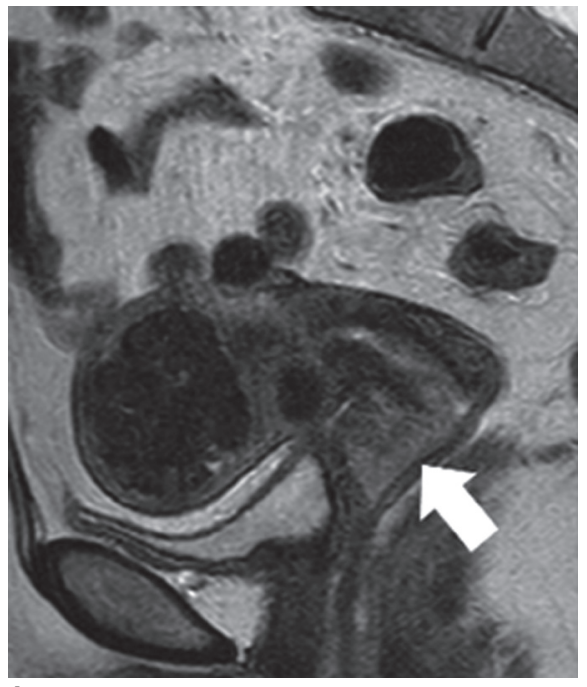

A

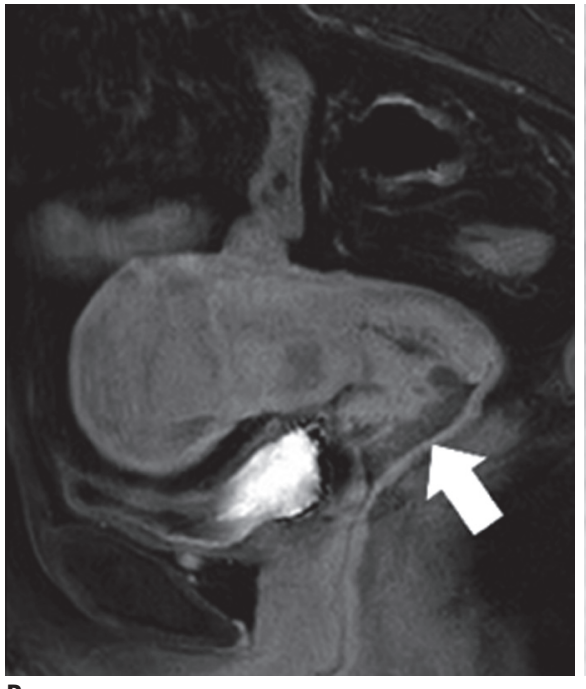

B

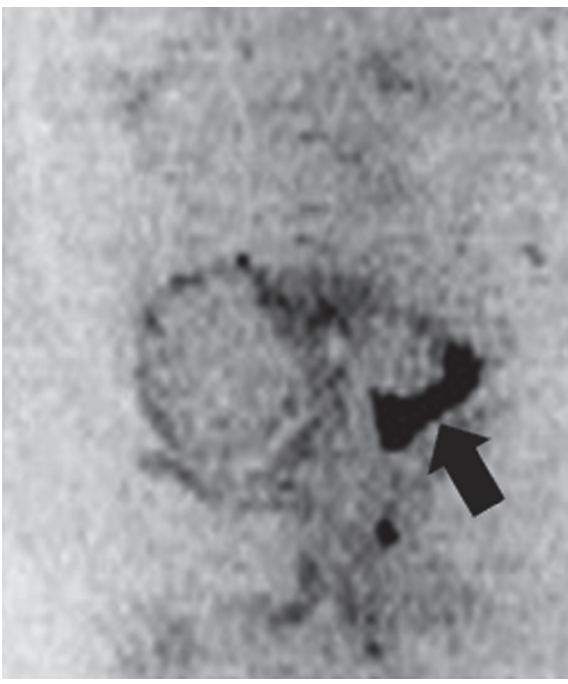

C

Fig. 2. 56-year-old woman with endocervical adenocarcinoma, usual type.

A. Sagittal T2WI shows exophytic tumor (protruding inside canal) in cervical lip (arrow) and indicates invasive solid pattern (Fig. 1; Type 4). This is typical pattern for usual type adenocarcinoma. B. Sagittal contrast-enhanced fat-saturated T1WI shows weak enhancement of tumor (arrow). C. Sagittal DWI shows marked restricted diffusion (arrow). DWI = diffusion-weighted imaging, T1WI = T1-weighted imaging, T2WI = T2-weighted imaging 

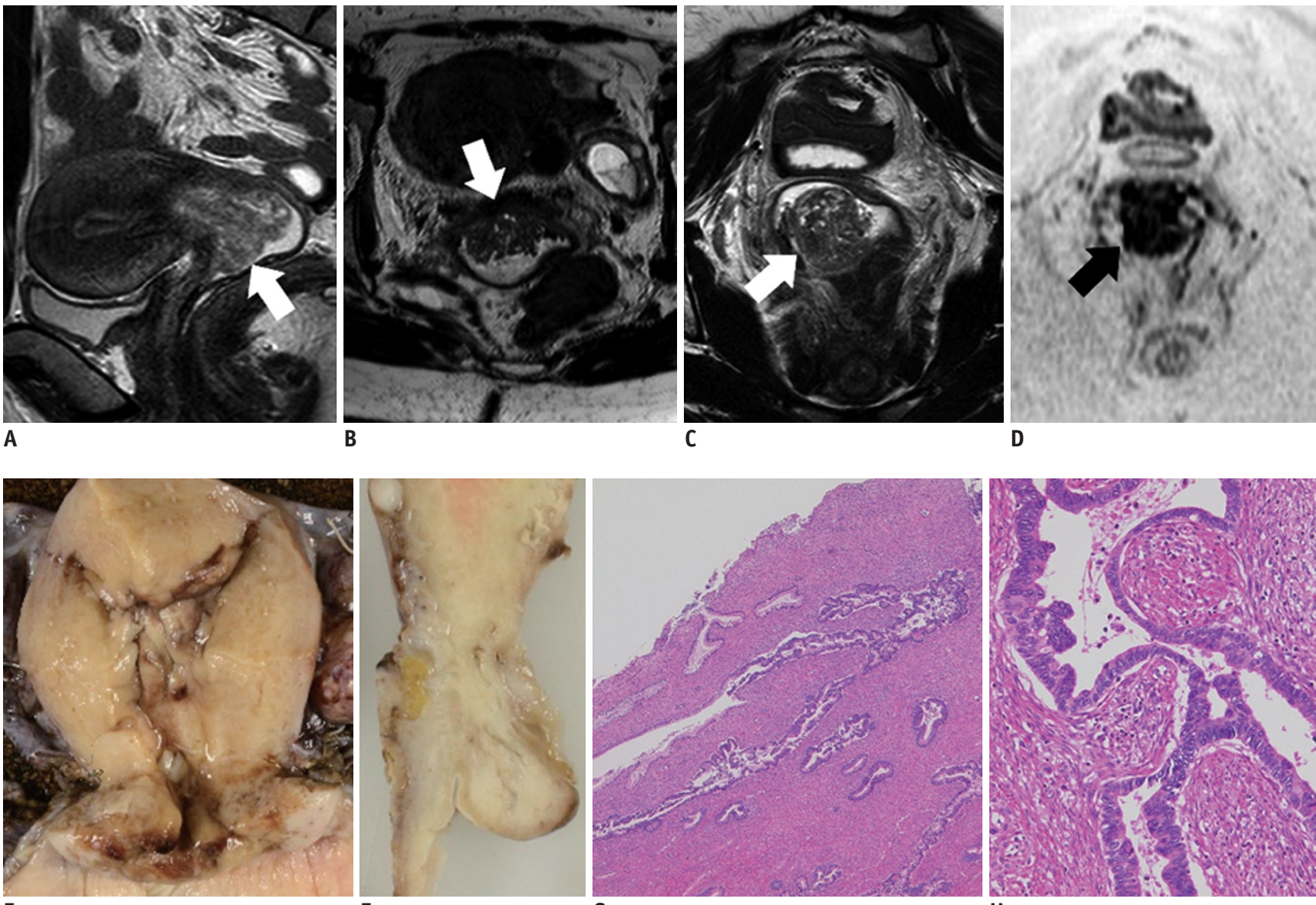

G

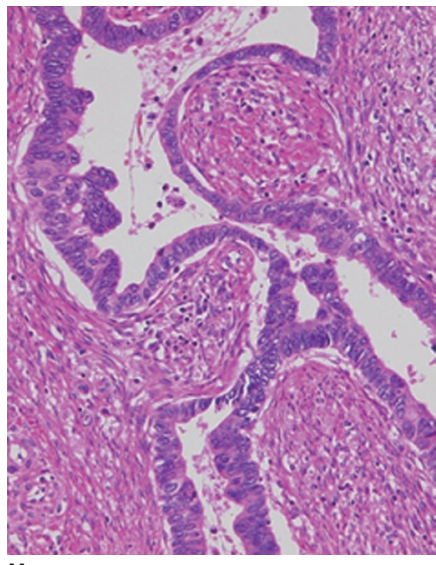

\section{Fig. 3. 30-year-old woman with endocervical adenocarcinoma, usual type.}

A. Sagittal T2WI shows exophytic tumor (arrow) in uterine cervix. Axial (B) and coronal (C) T2WIs show similar to fern leaf-like appearance, i.e., exophytic villous pattern (arrows) (Fig. 1; Type 2). D. Coronal DWI shows restricted diffusion (arrow). E, F. Fixed samples (E, coronal; F, sagittal) show diffuse enlargement of cervix, with invasive solid and diffuse infiltrative pattern, not exophytic villous pattern. G, H. On microscopic examination (G, hematoxylin-eosin staining, original magnification, $\times 40 ; \mathbf{H}$, hematoxylin-eosin staining, original magnification, $\times 200)$, tumor shows papillary surface, although it is composed of stromal hyperplasia caused by inflammation. Tumor cell glandules exist in inner part and show concave shape. Pseudostratification and necrotic debris within gland space are typical features of endocervical adenocarcinoma, usual type. Coexistence with CIN also indicates correlation with human papilloma virus infection. CIN = cervical intraepithelial neoplasia

An invasive solid pattern or a diffuse infiltrative pattern is commonly observed on MRI (Fig. 1; Types 4, 5), and it is generally difficult to distinguish these tumors from SCC using MRI alone. Sometimes, intratumoral cysts can be seen, but these are not common (11). The incidence of ovarian metastasis is higher with this type of adenocarcinoma than with SCC (12).

\section{Mucinous Carcinoma, Gastric Type (Figs. 4, 5)}

This is a mucinous adenocarcinoma that shows gastric type differentiation. Mucinous carcinoma gastric type (GAS) accounts for approximately $25 \%$ of all adenocarcinomas. GAS is not associated with high-risk HPV infection (Table 1), so its incidence threatens to increase despite improved screening programs. The mean age at presentation is approximately 42 years and its prognosis is poorer than that of the usual type adenocarcinoma. The 5-year diseasefree survival rate is $30 \%$, which is much lower than that of the usual type adenocarcinoma $(74 \%)(1,4)$. Its association with Peutz-Jeghers syndrome has been noted $(1,4)$. GAS includes minimal deviation adenocarcinoma (adenoma malignum). In addition, lobular endocervical glandular hyperplasia (LEGH), which is described later, is considered a precursor lesion of GAS according to recent studies (1, 4, 13). Immunohistochemical studies are positive for makers of pyloric gland mucins (eg., MUC6, HIK1083) (4).

GAS typically appears as a multicystic lesion indicating LEGH with inner solid components on MRI, namely a solid and cystic pattern (Fig. 1; Type 1). Diffuse, solid low T2weighted signal intensities with an unclear margin in 

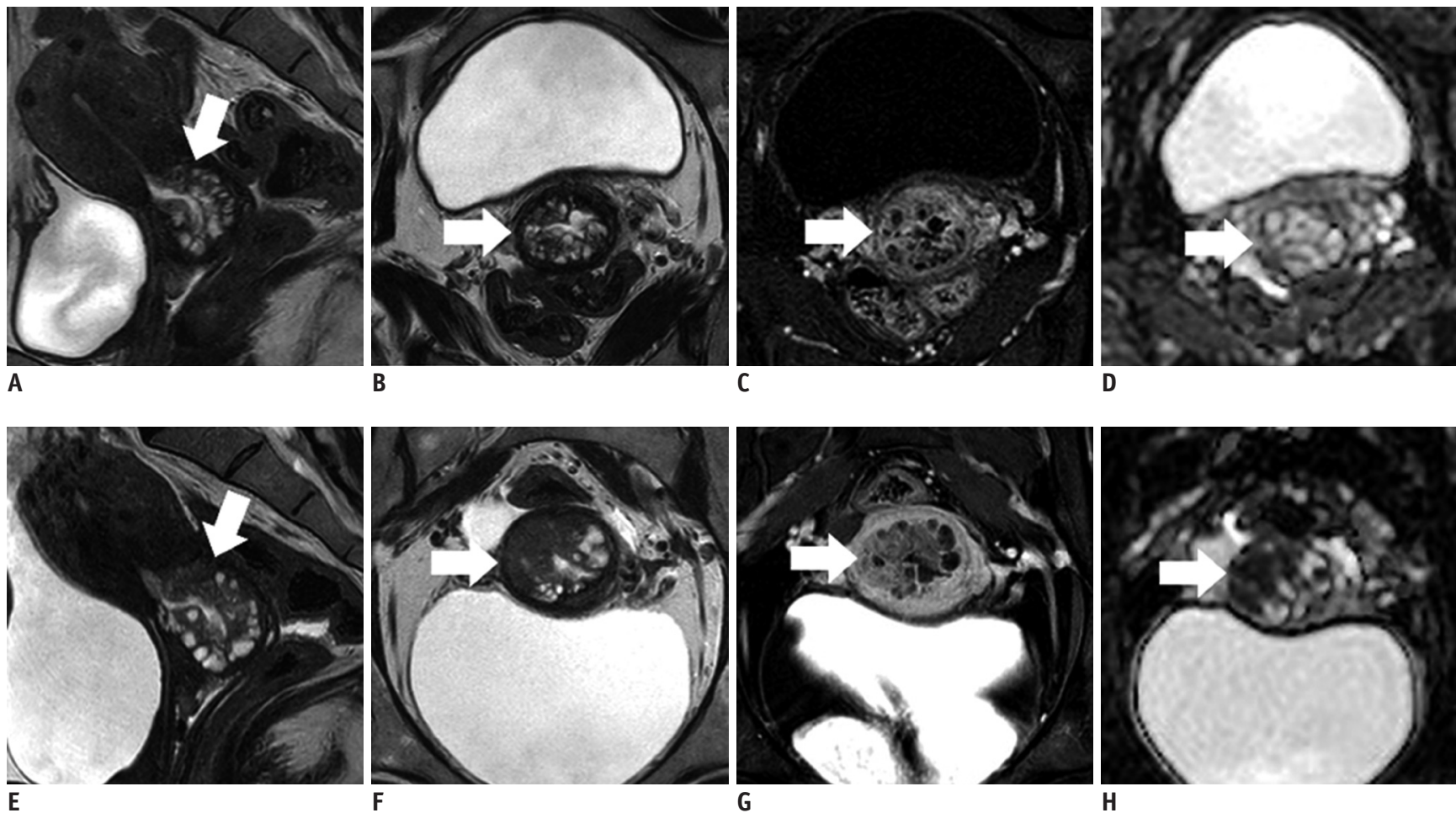

H
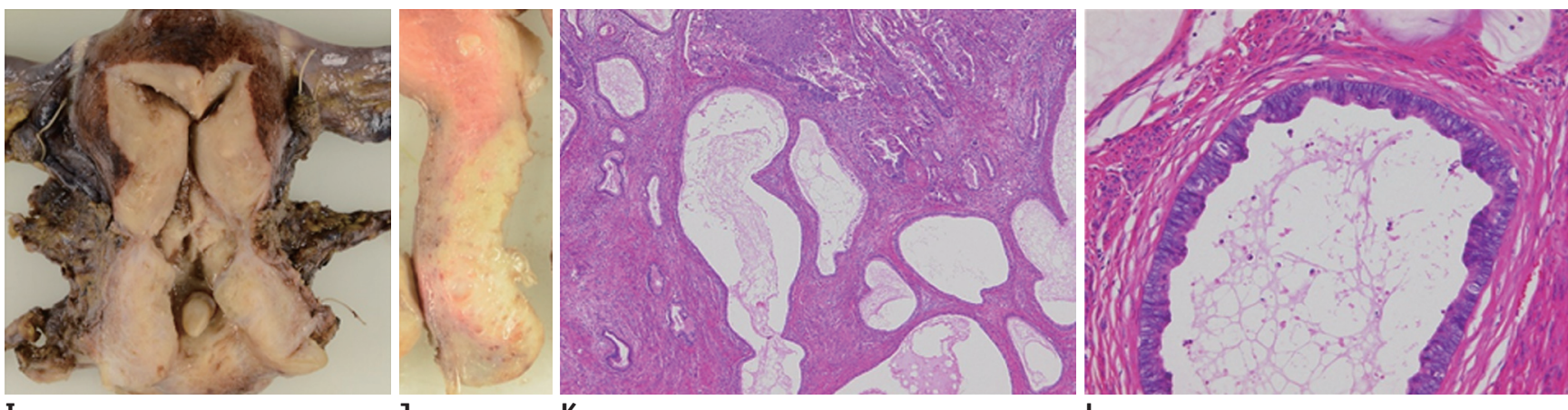

I

J

K

L

Fig. 4. 46-year-old woman with GAS (A-D: 1 year prior).

A, E. Sagittal T2WIs show multicystic mass with solid components (arrows) that increases in size. B, F. Axial T2WIs show solid components that have increased in size over year (arrows). Solid and cystic (Fig. 1; Type 1) as well as invasive solid patterns (Fig. 1; Type 4) are seen in this tumor. C, G. Axial contrast-enhanced fat-saturated T1WIs show mild enhancement of solid components (arrows). D, H. Restricted diffusion becomes clear on axial ADC map (arrows) over a year. I, J. Fixed samples (I, coronal; J, sagittal) show solid tumor with multiple cystic components. K. Microscopic examination (hematoxylin-eosin staining, original magnification, $\times 40$ ) shows tumor cells diffusely infiltrating and involving glandules of LEGH lesion, while maintaining its glandular cavities. L. Microscopic examination (hematoxylin-eosin staining, original magnification, $\times 200$ ) shows tumor cells with abundant clear cytoplasm. p16 negativity indicated lack of correlation with human papilloma virus infection (not displayed), which is characteristic of GAS. ADC = apparent diffusion coefficient, LEGH = lobular endocervical glandular hyperplasia

LEGH, or a solid component with restricted diffusion in LEGH, suggests an occurrence of GAS $(11,13)$; however, sometimes, solid components are small, lack of restricted diffusion, and are difficult to distinguish from LEGH (13). In addition, tumors often diffusely infiltrate and invade more widely than expected on MRI.

\section{Mucinous Carcinoma, Intestinal Type (Fig. 6)}

This is a mucinous adenocarcinoma that shows area of intestinal-type differentiation. It accounts for about $2 \%$ of all cervical adenocarcinoma $\mathrm{s}$ and is associated with high-risk HPV infection (Table 1). Occasionally, the usual type adenocarcinoma coexists with this tumor depending on its association with high-risk HPV infection $(1,4)$. The intestinal differentiation shows typical mucinous-type epithelium and the most important differential diagnosis is 


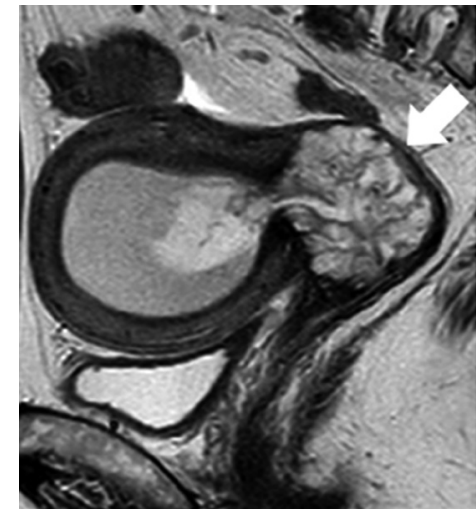

A

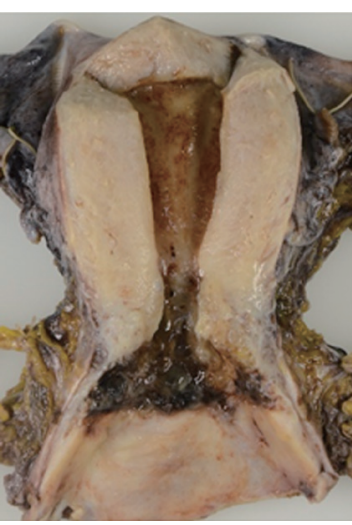

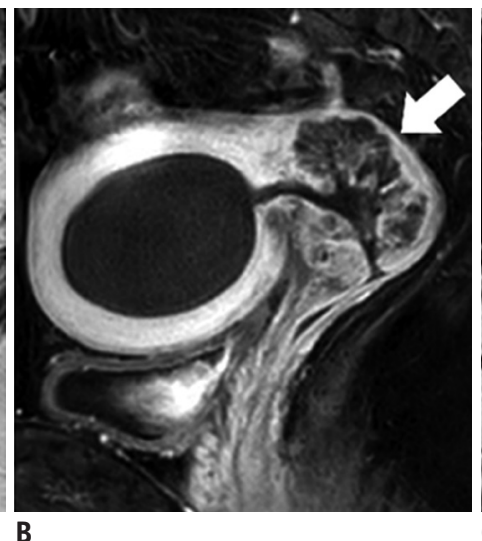
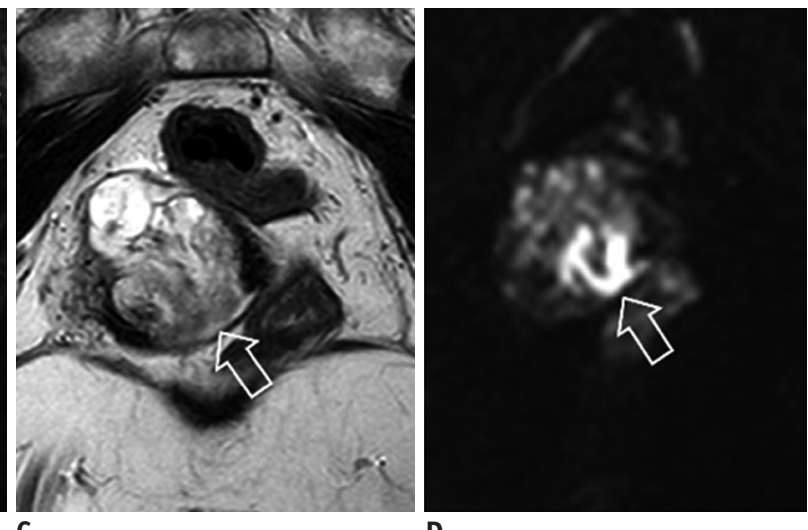

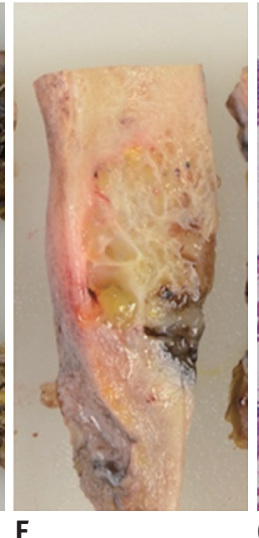

$\mathbf{F}$
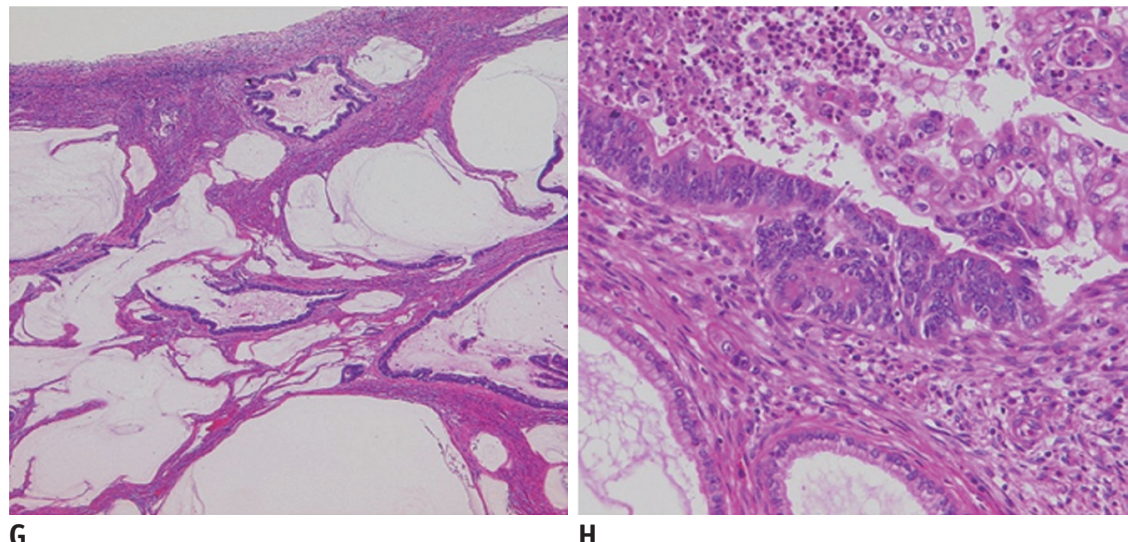

Fig. 5. 56-year-old woman with GAS.

A. Sagittal T2WI shows multicystic mass (arrow) in uterine cervix, consistent with Type 1 appearance (Fig. 1). B. Sagittal contrast-enhanced fatsaturated T1WI shows well enhanced septa in multicystic mass (arrow). C. Axial T2WI shows core-like hypointense lesion in center of multicystic mass (void arrow). D. Axial DWI shows restricted diffusion in solid core (void arrow), and solid component with restricted diffusion suggests GAS occurring in LEGH lesion. E, F. Fixed samples (E, coronal; F, sagittal) show multicystic tumor in uterine cervix. G, H. Microscopic examination (G, hematoxylin-eosin staining, original magnification, x 40; H, hematoxylin-eosin staining, original magnification, x 200) shows tumor composed of abundant mucinous lakes. Cluster of tumor cells exist in core, and tumor cells are widely spread to line mucinous lakes. p16 negativity indicated lack of correlation with human papilloma virus infection (not displayed).

metastasis from intestinal carcinoma. Immunohistochemical studies are positive for makers of intestinal mucins (e.g., CDX2) (4).

An invasive solid pattern or a diffuse infiltrative pattern is commonly observed on MRI (Fig. 1; Types 4, 5), and it generally shows relatively high signal intensities on T2weighted imaging (T2WI) and weak restricted diffusion reflecting intracellular mucinous components (14).

\section{Villoglandular Carcinoma (Fig. 7)}

This is a variant of endocervical adenocarcinoma that shows a distinct exophytic, villous-papillary growth. It accounts for $3.7-4.8 \%$ of all cervical adenocarcinomas, and associated with high-risk HPV infection (Table 1 ). It affects younger individuals, and the mean age at presentation is approximately 35 years $(1,4)$. It has a good prognosis and fertility-preserving surgery may be considered if the tumor is in the early stage $(1,15)$. 0ccasionally, CIN or AIS coexists with this tumor depending on its association with high-risk HPV infection (1).

A fern leaf-like appearance, i.e., an exophytic villous pattern (Fig. 1; Type 2), with restricted diffusion is a typical MRI finding of villoglandular carcinoma (VGC) (16).

\section{Clear Cell Carcinoma (Fig. 8)}

This adenocarcinoma is predominantly composed of clear or hobnail cells with solid, tubulocystic, and/or papillary architectural patterns. Microscopically, a solid tumor typically contains abundant glycogen-rich cytoplasm. It accounts for $2-4 \%$ of all cervical adenocarcinomas, and not associated with high-risk HPV infection (Table 1) $(4,5)$. In utero diethylstilbestrol exposure causes clear cell carcinoma in young patients with an average age of 19 years. However, the tumor now becomes rare, and most cases are sporadic 


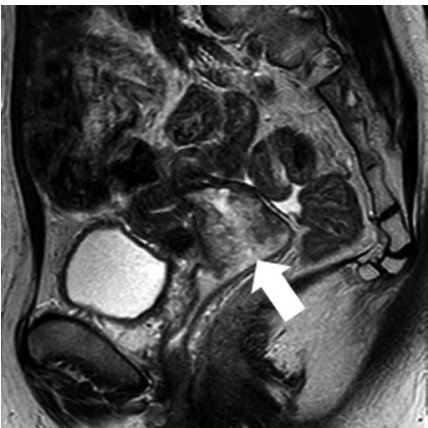

A

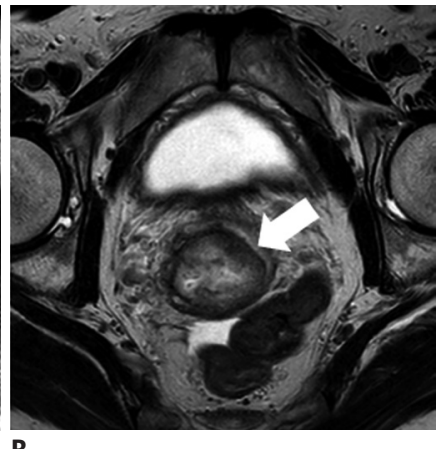

B

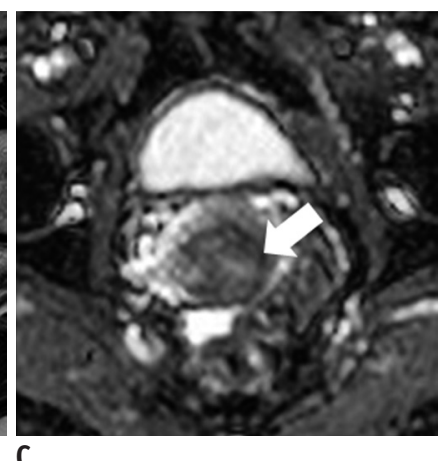

C

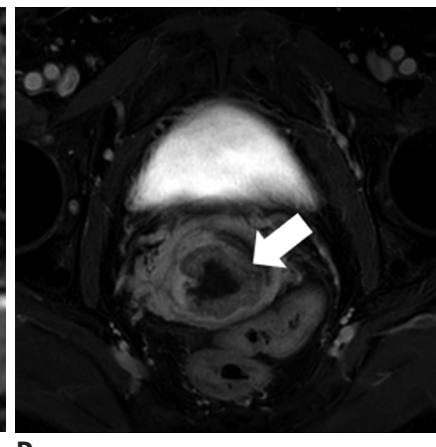

D

Fig. 6. 57-year-old woman with mucinous carcinoma, intestinal type.

Sagittal (A) and axial (B) T2WIs show high signal-intensity mass with invasive solid pattern (Fig. 1; Type 4) in uterine cervix (arrows). C. Tumor shows weak restricted diffusion on axial ADC map (arrow). Relatively high signal intensities on T2WI and weak restricted diffusion seem to reflecting intracellular mucinous components. D. Tumor is mildly enhanced on axial contrast-enhanced fat-saturated T1WI (arrow).

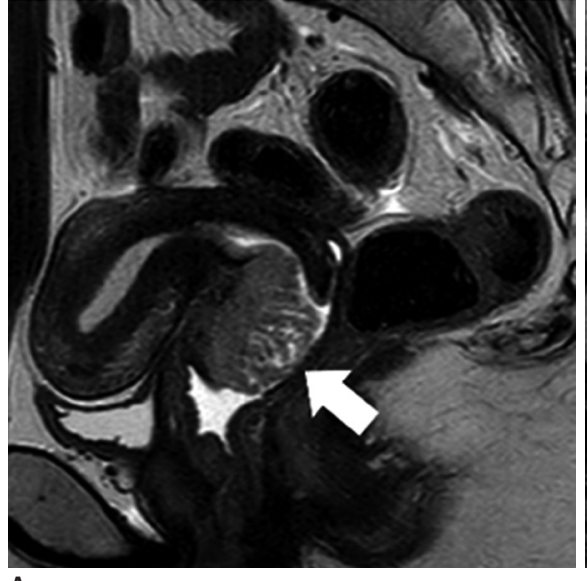

A

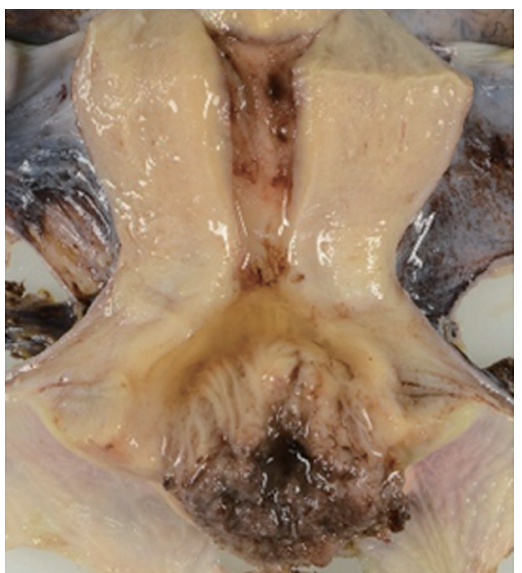

D

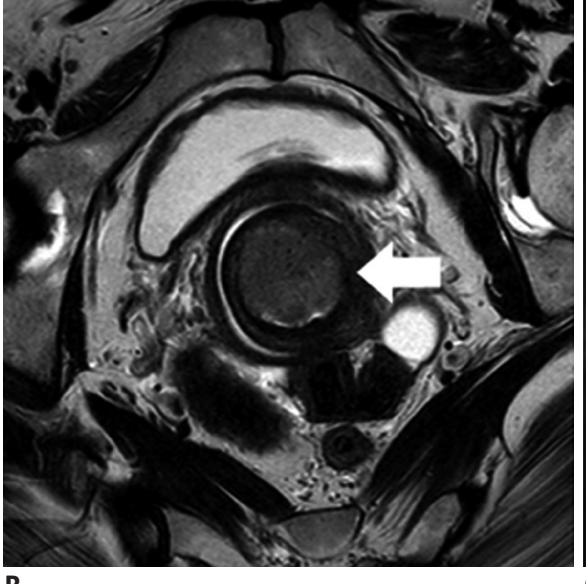

B

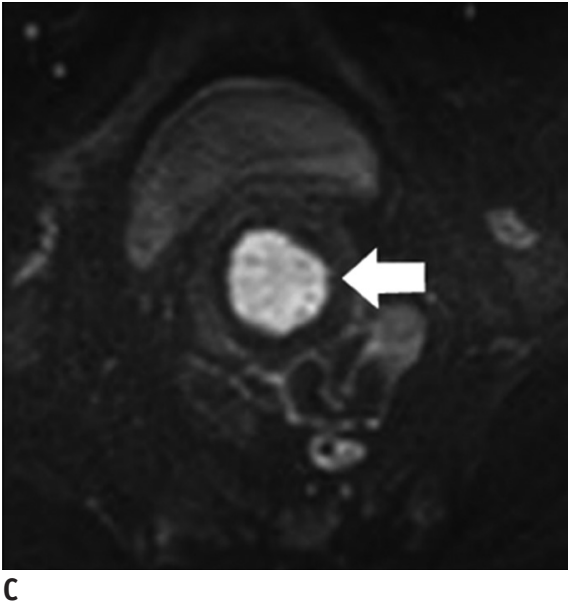

C

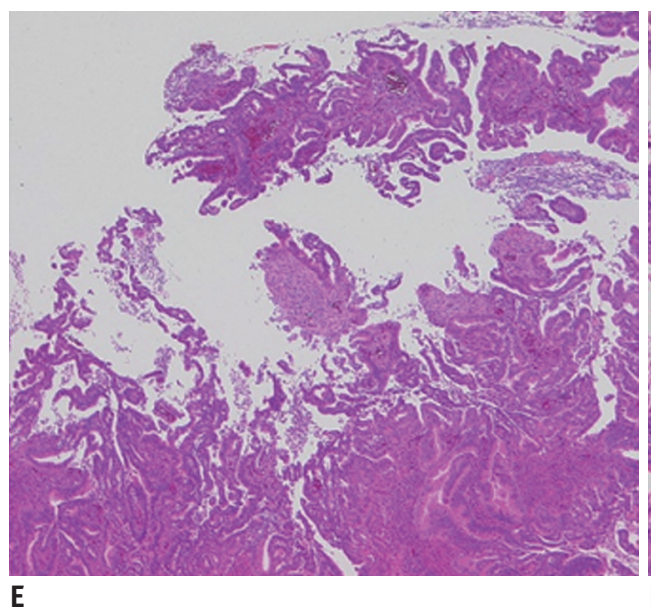

E

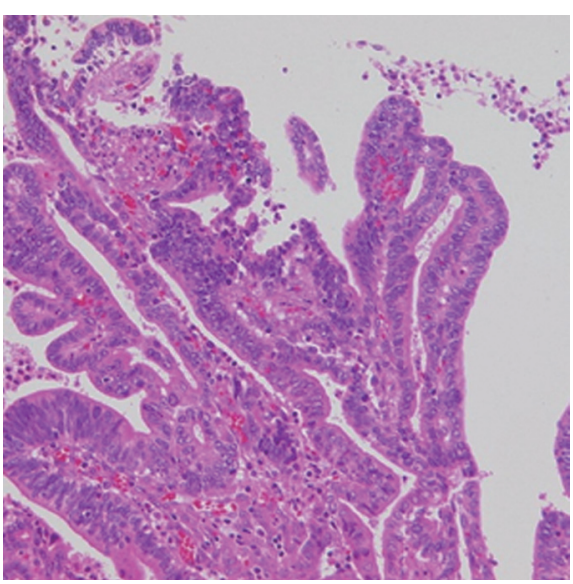

$\mathbf{F}$

Fig. 7. 36-year-old woman with villoglandular carcinoma.

Sagittal (A) and axial (B) T2WIs show cervical tumor with fern leaf-like appearance (exophytic villous pattern, Fig. 1; Type 2) (arrows). C. On axial DWI, tumor shows restricted diffusion (arrow), and malignancy is suggested. D. Fixed sample shows cervical tumor with fern leaf-like appearance. E, F. Microscopic examination (E, hematoxylin-eosin staining, original magnification, x 40; F, hematoxylin-eosin staining, original magnification, $\times 20$ ) shows exophytic tumor with villous fronds lined by well-differentiated tumor cells. Coexistence with CIN also indicates correlation of this tumor with human papilloma virus infection. 


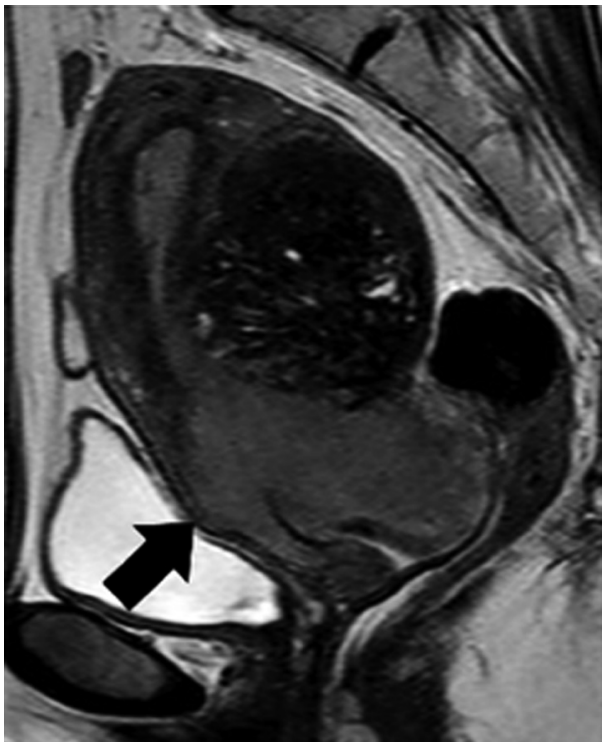

A

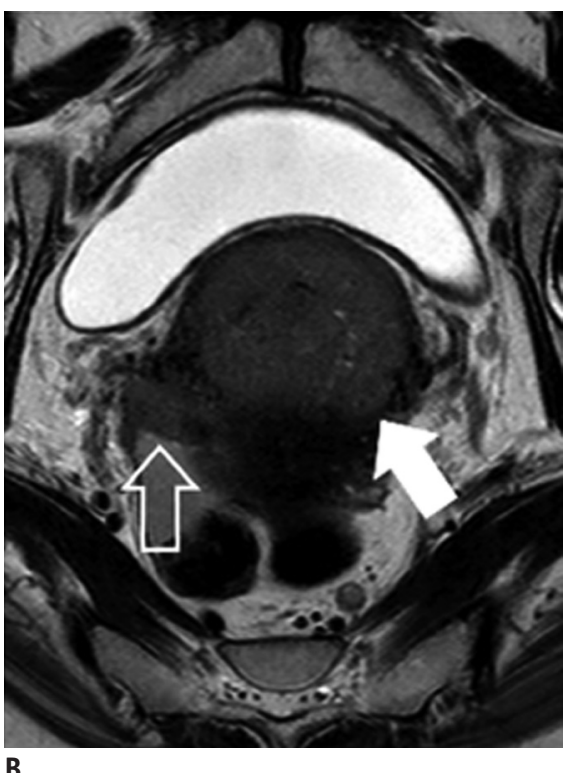

B

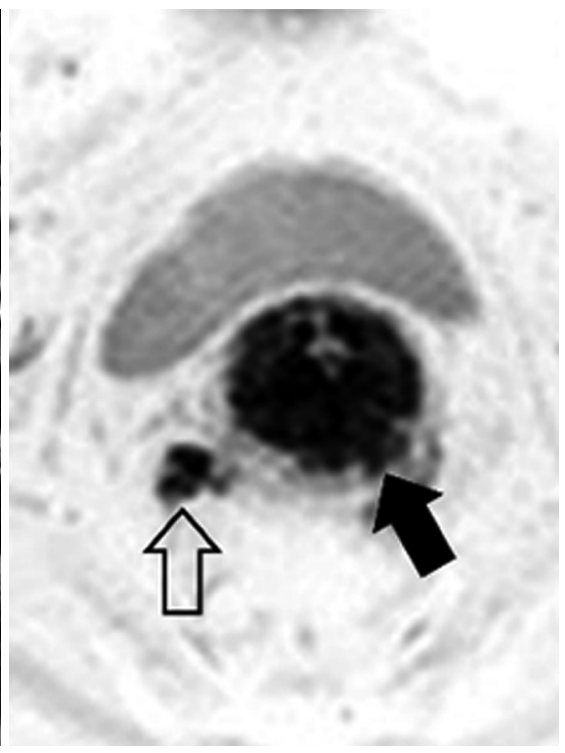

C

Fig. 8. 53-year-old woman with clear cell carcinoma.

Sagittal (A) and axial (B) T2WIs show invasive solid tumor (Fig. 1; Type 4) in uterine cervix (arrows) with right parametrial invasion reaching pelvic wall (void arrow). C. On axial DWI, tumor (arrow) and parametrical invasion (void arrow) both show restricted diffusion. It is difficult to distinguish clear cell carcinoma from SCC and endocervical adenocarcinoma, usual type, by MRI alone.

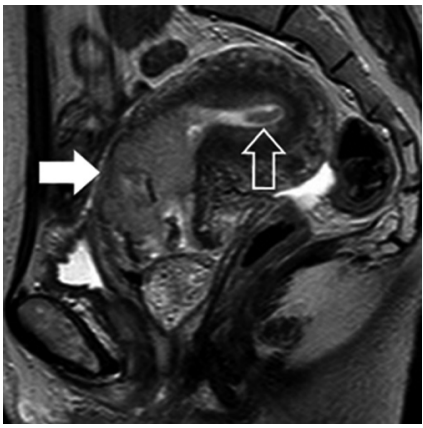

A

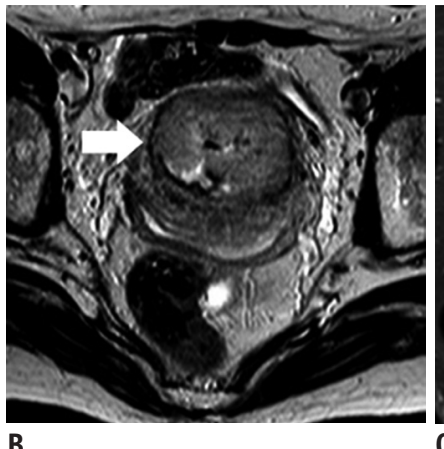

B
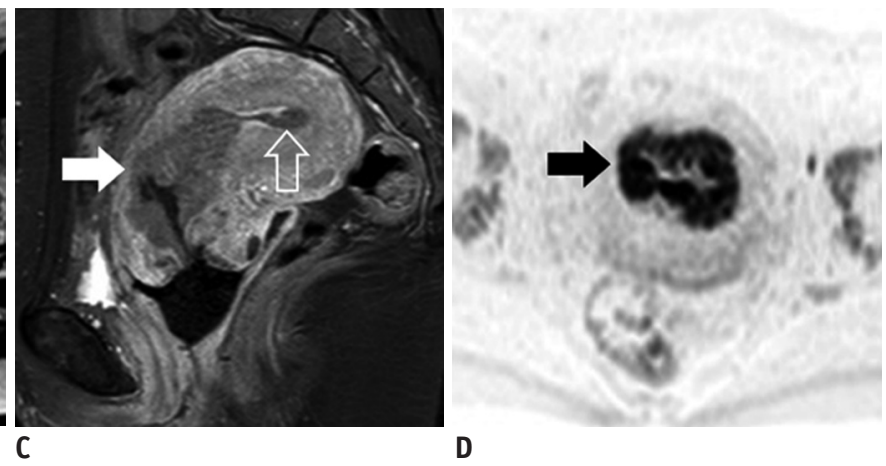

Fig. 9. 47-year-old woman with endometrioid carcinoma

Sagittal (A) and axial (B) T2WIs show invasive solid tumor (Fig. 1; Type 4) mainly located in cervical canal (arrows). Small tumor is also present in uterine corpus (void arrow). C. Sagittal contrast-enhanced fat-saturated T1WI shows mild enhancement of both tumors (arrow, void arrow). D. On axial DWI, tumor shows restricted diffusion (arrow). Pathological diagnosis was uterine corpus carcinoma, stage II, but final clinical diagnosis, which was based on main tumor location, was uterine cervical endometrioid carcinoma, stage IB2, with dissemination in uterine body cavity.

and involve older patients with an average age of 47 years (1). Clear cell carcinoma is clinically similar to the usual type adenocarcinoma, and its prognosis is also similar to that of the usual type adenocarcinoma (1).

It is difficult to distinguish clear cell carcinoma from SCC and the usual type adenocarcinoma using MRI alone (Fig. 1).

\section{Endometrioid Carcinoma (Fig. 9)}

This adenocarcinoma arises in the cervix and exhibits endometrioid morphological features (1) and is not associated with high-risk HPV infection (Table 1) $(4,5)$. It is clinically and macroscopically similar to the usual type adenocarcinoma, and some researchers suggest that it is just a morphological variant of the usual type adenocarcinoma (1).

Usually, it is difficult to distinguish endometrioid carcinoma from SCC and the usual type adenocarcinoma using MRI alone (Fig. 1). Moreover, the most important differential diagnosis is corpus endometrioid carcinoma extending to the cervix, which can be clinically misdiagnosed as cervical endometrioid carcinoma (1).

\section{Other Cervical Tumors and Tumor-Like Lesions}

Lobular Endocervical Glandular Hyperplasia (Fig. 10)

This is a distinct benign lobular proliferation of 
endocervical glands that commonly exhibits gastric/pyloric differentiation. Pyloric gland metaplasia is a synonym for LEGH. LEGH is considered a potential precursor lesion of GAS, and GAS or AIS occasionally coexists in LEGH lesions, necessitating careful attention in diagnosis. It may occur in women of reproductive age or postmenopausal women and watery or mucoid discharge is a characteristic clinical manifestation (1).

Typically, it is located in the upper cervical canal. MRI findings of LEGH show two patterns, a cosmos pattern and a microcystic pattern. The cosmos pattern is characterized by central microcystic components surrounded by medium-sized to large cysts, while the microcystic pattern is characterized by the aggregation of small cysts without peripheral larger cysts. LEGH, particularly the microcystic pattern type, may be sometimes difficult to distinguish from GAS or AIS using MRI alone (13).

In addition, other cystic lesions need to be differentiated from LEGH, such as Nabothian cyst, tunnel cluster, and endocervical polyp $(13,17)$, all of which are categorized as benign glandular tumors and tumor-like lesions in the fourth edition of the World Health Organization (WHO) classification and not associated with high-risk HPV infection (Table 1) (1).

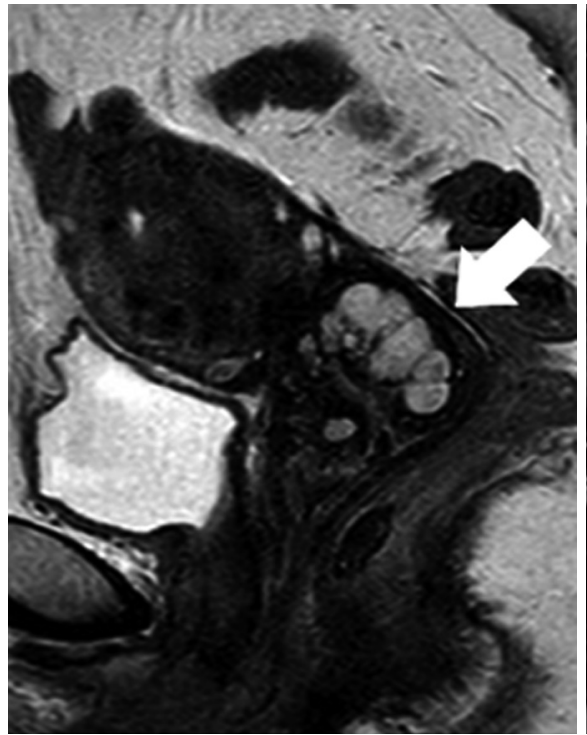

A

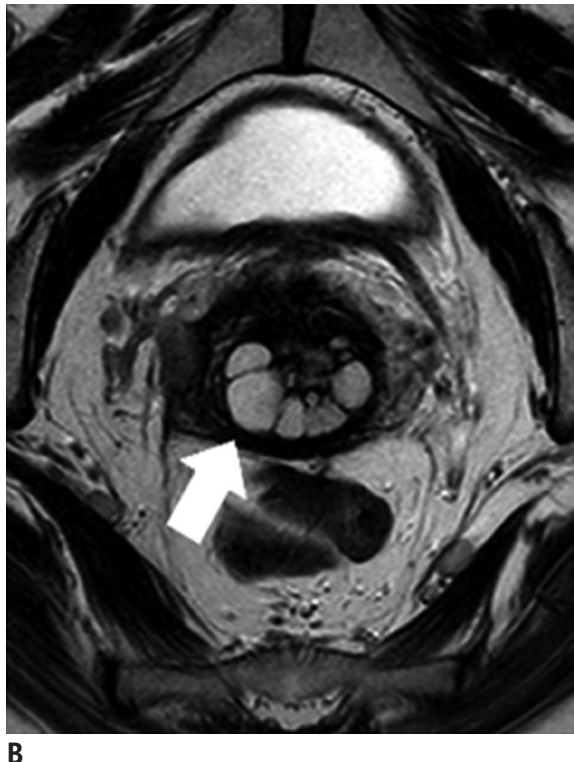

B

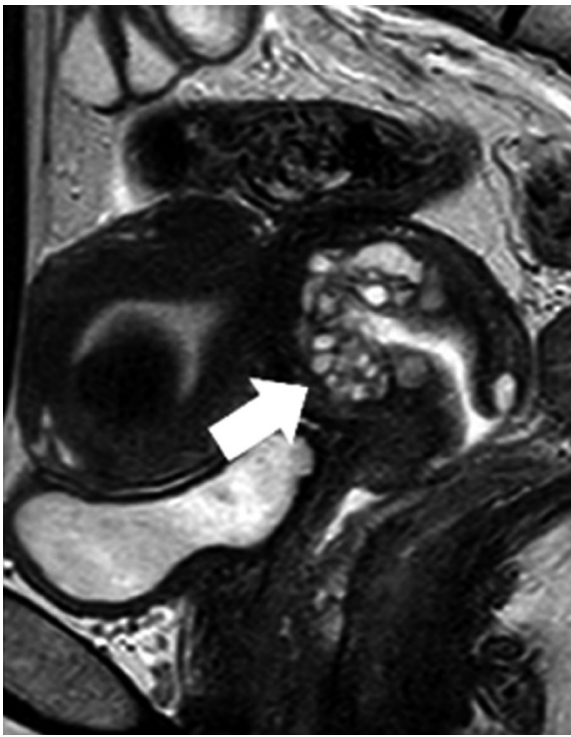

C

Fig. 10. 45-year-old woman with cosmos pattern LEGH (A, B) and 47-year-old woman with microcystic pattern LEGH (C). Sagittal (A) and axial (B) T2WIs show multicystic lesion in uterine cervix (arrows) with Type 1 appearance (Fig. 1). Microcytic parts surrounded by medium-sized to large cysts, indicating cosmos pattern. C. Sagittal T2WI shows multicystic lesion in uterine cervix (arrow) (Fig. 1; Type 1). Aggregation of small cysts without peripheral large cysts, indicating microcystic pattern.

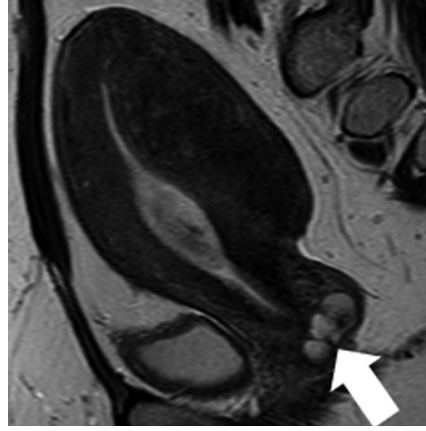

A

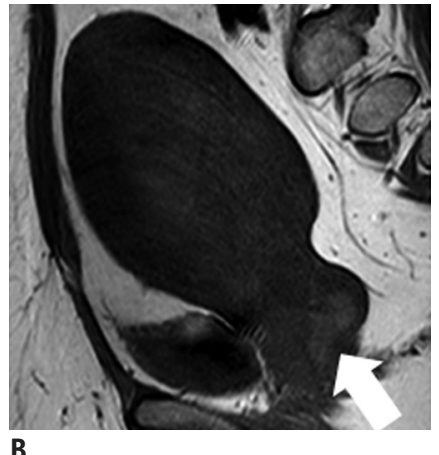

B
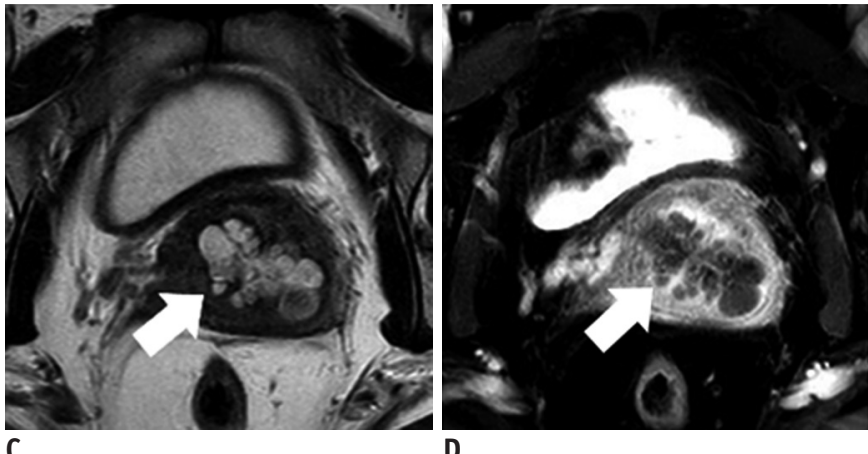

D

Fig. 11. 45-year-old woman with Nabothian cyst.

A. Sagittal T2WI shows multicystic lesion near surface of cervical lip (arrow). B. On axial T1WI, cysts show slightly high signal intensity (arrow). C. On axial T2WI, cysts show high to intermediate signal intensity (arrow). Intermediate signal intensities on T2WI and slightly high signal intensities on T1WI indicate mucus contents, and this is characteristic imaging finding of Nabothian cysts. D. There is no enhanced solid component in cysts (arrow) on axial contrast-enhanced fat-saturated T1WI. 


\section{Nabothian Cyst (Fig. 11)}

This is a cystic structure in the cervical wall that is lined by normal endocervical epithelium and filled with mucus. A Nabothian cyst is a common finding, particularly in multiparous women (1). It is usually asymptomatic and often found incidentally on MRI.

These cysts may be single or multiple, and they usually measure $2-10 \mathrm{~cm}$ in diameter. Generally, they are located near the surface of the endocervical canal, but they are occasionally found deep in the endocervical wall (1). The cyst is filled with mucus and characteristically exhibits high to intermediate signal intensities on T2WI and intermediate or slightly high signal intensities on T1-weighted imaging (17).

\section{Endocervical Polyp (Fig. 12)}

This is an exophytic lesion lined by benign endocervical epithelium covering a fibrovascular core. Sometimes a focus of in situ or invasive carcinoma can be contained within an endocervical polyp. It is one of the most common causes of intermenstrual vaginal bleeding (1).

A multicystic structure with a fibrovascular core is a characteristic MRI finding (17).

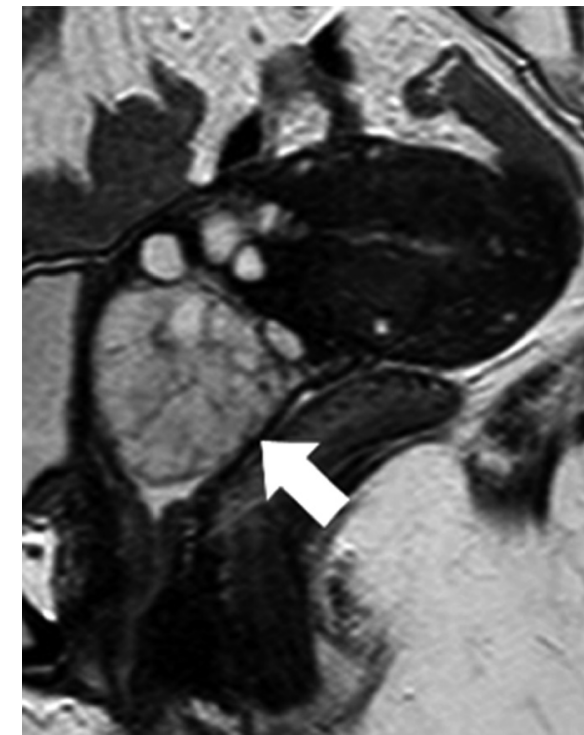

A

Fig. 12. 53-year-old woman with cervical polyp.

Sagittal (A) and axial (B) T2WIs show exophytic, oval, multicystic lesion protruding from cervix into vagina (arrows). C. Sagittal contrastenhanced fat-saturated T1WI shows well-enhanced septa in multicystic lesion (arrow). Fibrovascular core is not clear; therefore, it is difficult to distinguish cervical polyp from LEGH in this case.
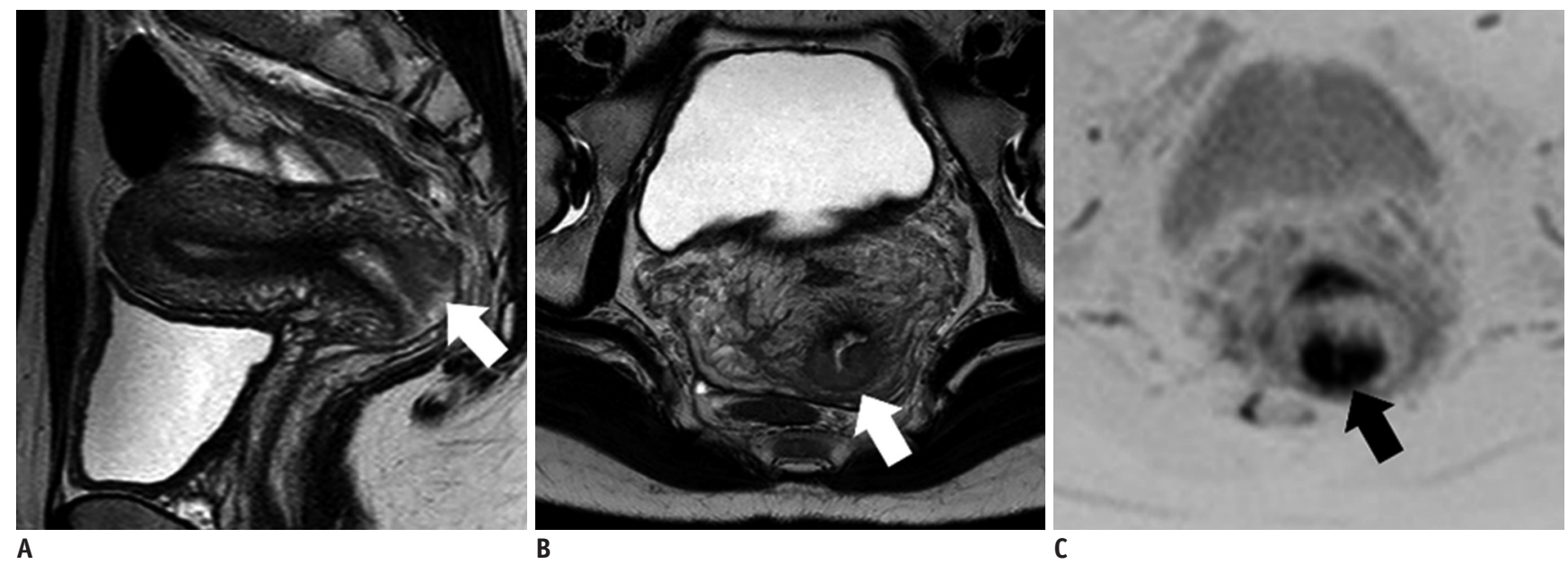

Fig. 13. 30-year-old woman with ASC.

Sagittal (A) and axial (B) T2WIs show invasive solid tumor (Fig. 1; Type 4) located in posterior cervical lip (arrows). C. Axial DWI shows markedly restricted diffusion (arrow). It is difficult to distinguish ASC from SCC and endocervical adenocarcinoma, usual type. 


\section{Adenosquamous Carcinoma (Fig. 13)}

This is a malignant tumor having both adenocarcinoma and SCC components, and the adenocarcinoma component should be adequately differentiated on the basis of histologically recognizable glands. Adenosquamous carcinoma (ASC) accounts for $3.6 \%$ of all cervical carcinomas, and usually be associated with high-risk HPV infection (Table 1) $(4,18)$. The mean age at presentation is approximately 50 years (6). Some studies have suggested a poorer prognosis than that of SCC and the usual type adenocarcinoma $(4,6,7)$.

It is generally difficult to distinguish this entity from SCC and the usual type adenocarcinoma using MRI alone (Fig. 1).

\section{Neuroendocrine Tumor (Fig. 14)}

This tumor is similar to gastro-entero-pancreatic neuroendocrine tumors (NETs) and is divided into highgrade and low-grade types. High-grade NETs are composed of high-grade malignant cells and may be either the small cell type or the large cell type. NETs are associated with high-risk HPV infection (Table 1), and may be accompanied by in situ or invasive SCC or adenocarcinoma. High-grade NETs are very aggressive, often accompanied by lymph node and distant metastasis and indicate a poor prognosis $(19,20)$.

MRI findings widely vary from the expansile solid pattern and the invasive solid pattern to the diffuse infiltrative pattern (Fig. 1; Types 3-5). Cervical NETs tend to appear as homogeneous lesions with multiple lymphadenopathies and are associated with a low apparent diffusion coefficient $(A D C)(17,21)$.

\section{Leiomyoma (Fig. 15)}

This is a benign tumor showing smooth muscle differentiation and containing a variable amount of collagen-rich extracellular matrix. It is categorized as a mesenchymal tumor and tumor-like lesion in the fourth edition of the WHO classification and not associated with high-risk HPV infection (Table 1) (1). Cervical leiomyoma is much rarer than leiomyoma involving the uterine body, and its frequency has been estimated to be $0.6 \%$ in hysterectomy specimens (22).

The lesion shows an expansile solid pattern (Fig. 1; Type 3) on MRI and typically appears as a well-defined, homogeneous, hypointense mass in the cervical stroma on T2WI, without restriction on diffusion-weighted imaging (DWI) $(17,23,24)$.

\section{Leiomyosarcoma (Fig. 16)}

This is a malignant tumor showing smooth muscle differentiation. Primary sarcomas account for less than $1 \%$ of all malignant cervical tumors, and leiomyosarcoma is the most common type (22) and not associated with high-risk HPV infection (Table 1) (1).

MRI shows an expansile solid pattern or an invasive

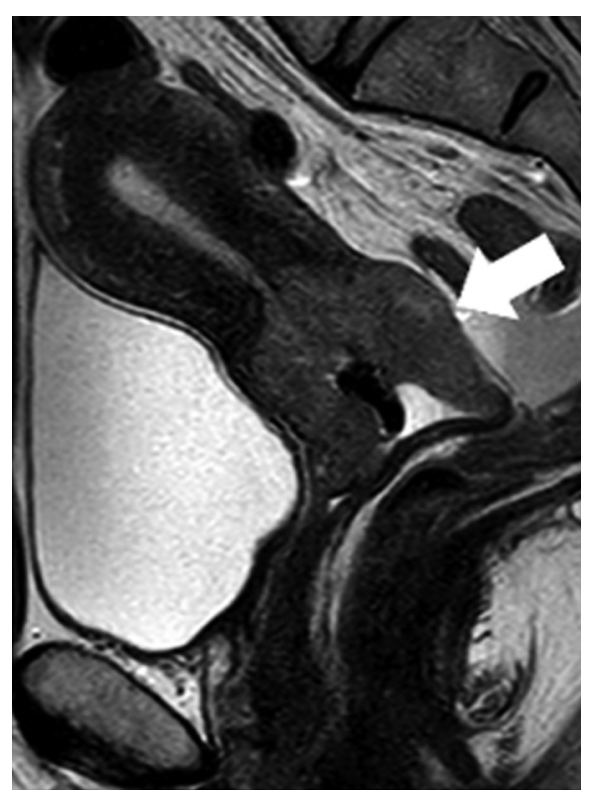

A

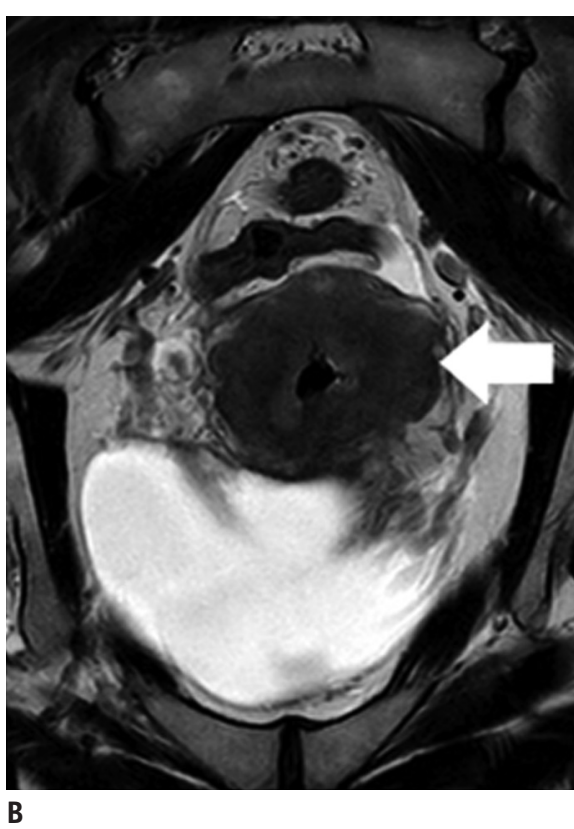

B

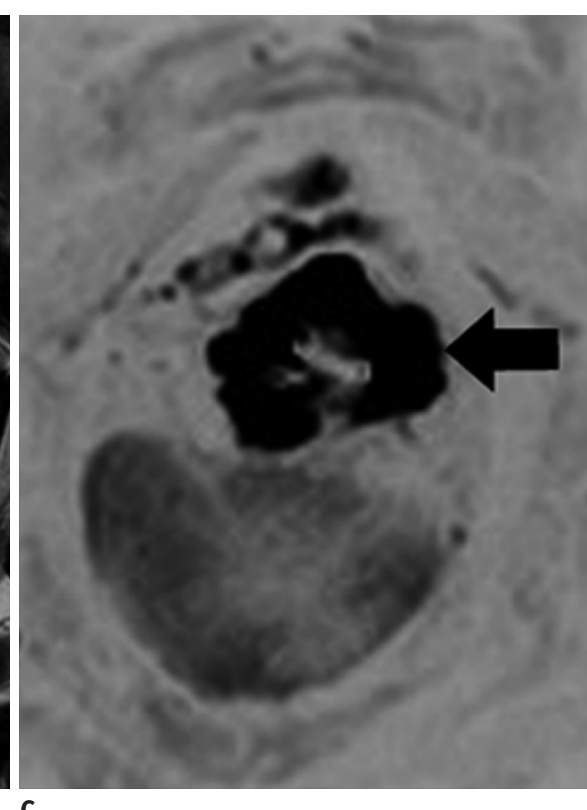

C

Fig. 14. 63-year-old woman with large cell neuroendocrine carcinoma.

Sagittal (A) and axial (B) T2WIs show invasive solid tumor (Fig. 1; Type 4) in uterine cervix (arrows). C. Axial DWI shows markedly restricted diffusion (arrow). This lesion is also difficult to distinguish from SCC and endocervical adenocarcinoma, usual type. 
solid pattern (Fig. 1; Types 3, 4). Leiomyosarcoma tends to show relatively high intensities on T2WI and restriction on DWI. Hemorrhage or necrosis and invasion of surrounding structures may be seen; these findings suggest leiomyosarcoma rather than leiomyoma $(17,23,24)$.

\section{Lymphoma (Fig. 17)}

Primary or secondary lymphoma is a malignant neoplasm composed of lymphoid cells without association of highrisk HPV infection (Table 1) (1). Uterine involvement is uncommon and primary lymphoma is much rarer.
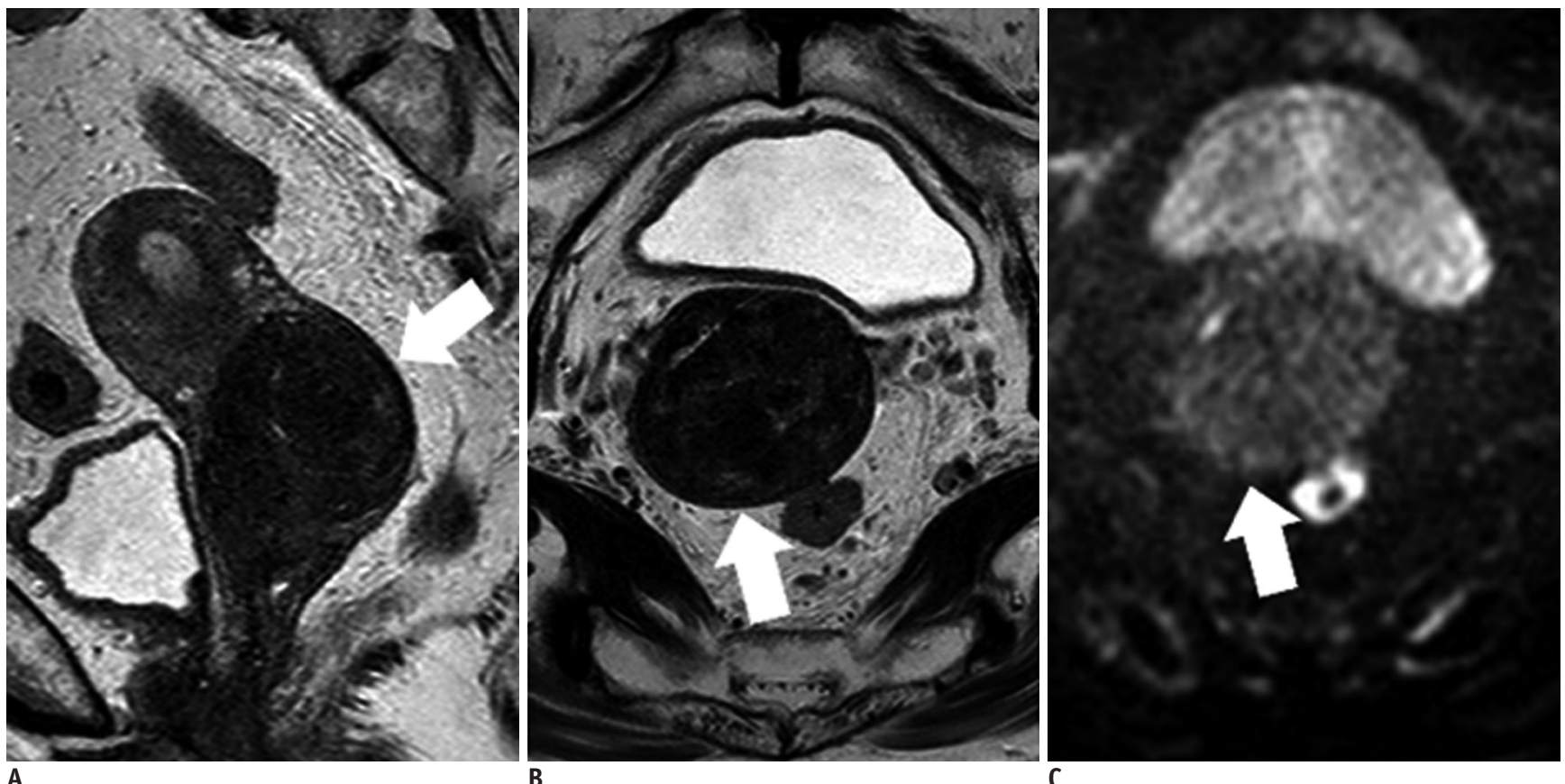

Fig. 15. 70-year-old woman with leiomyoma.

Sagittal (A) and axial (B) T2WIs show well-defined, homogeneous, hypointense mass (Fig. 1; Type 3) in stroma of uterine cervix (arrows). C. Axial DWI does not show restricted diffusion (arrow).

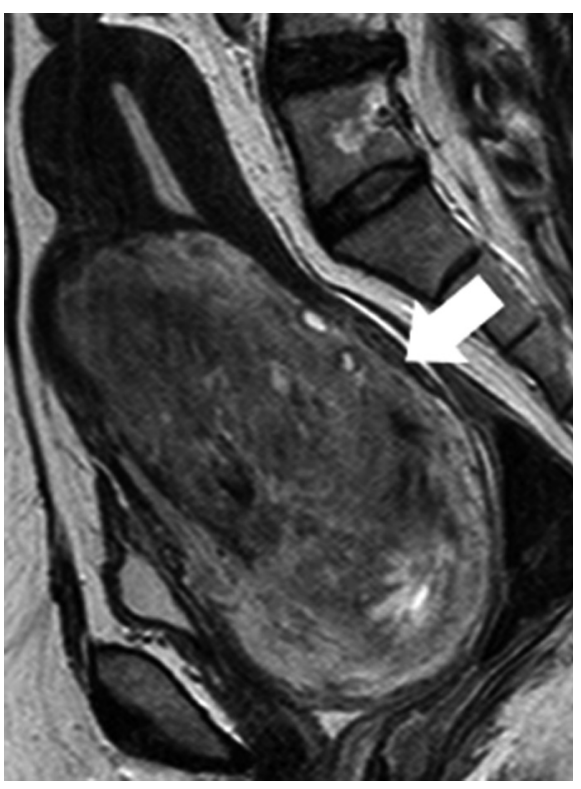

A

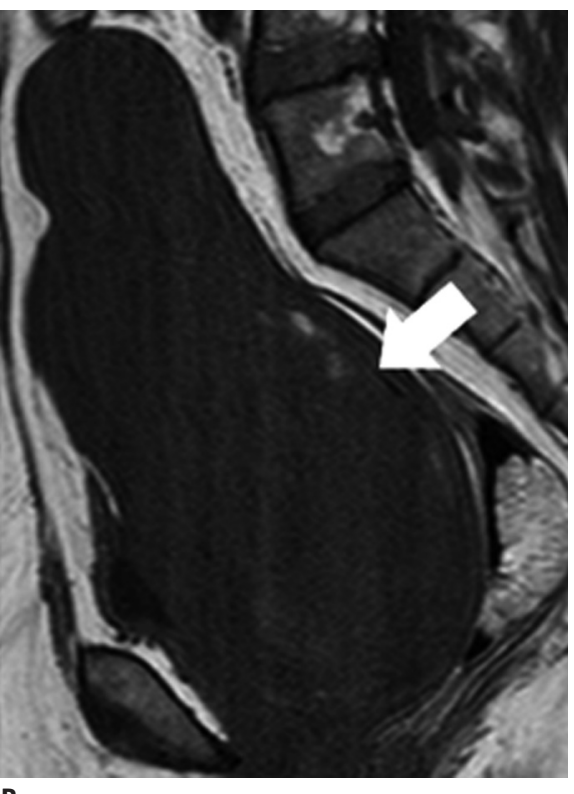

B

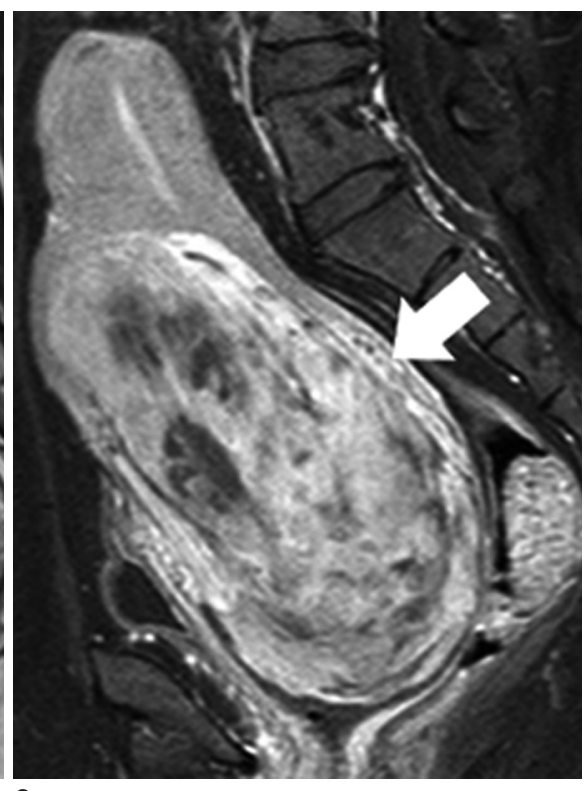

C

Fig. 16. 50-year-old woman with leiomyosarcoma.

A. Sagittal T2WI shows invasive solid tumor (Fig. 1; Type 4) in myometrium of uterine cervix (arrow), with relatively high signal intensity. B. Sagittal T1WI shows foci of high signal intensity in tumor (arrow), which are considered to be hemorrhagic foci. C. Heterogeneous enhancement is seen in tumor (arrow) on sagittal contrast-enhanced fat-saturated T1WI. Nonenhanced areas indicate tumor necrosis with hemorrhage. 
The majority of primary uterine lymphomas are B-cell lymphomas. The primary lesions commonly involve the cervix and usually respond well to chemotherapy and radiotherapy and exhibit a favorable prognosis, while the secondary lesions involve either the corpus or the cervix and has worse prognosis $(25,26)$.

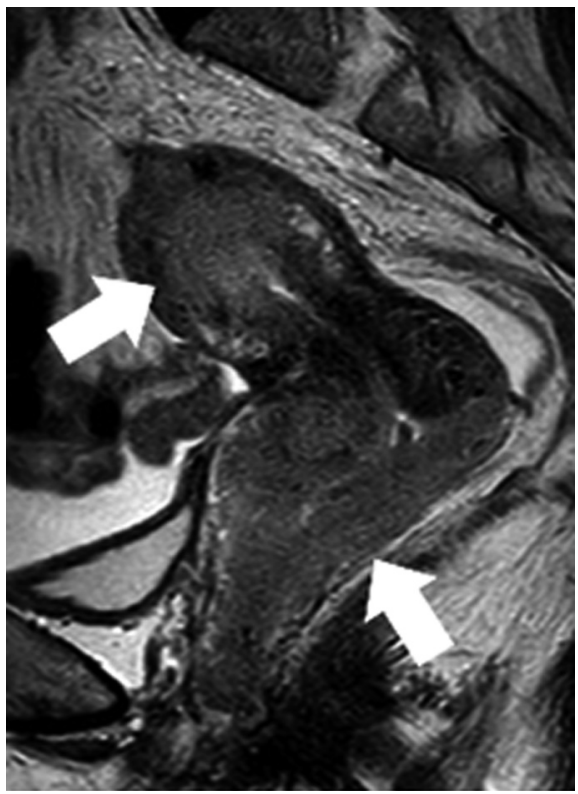

A

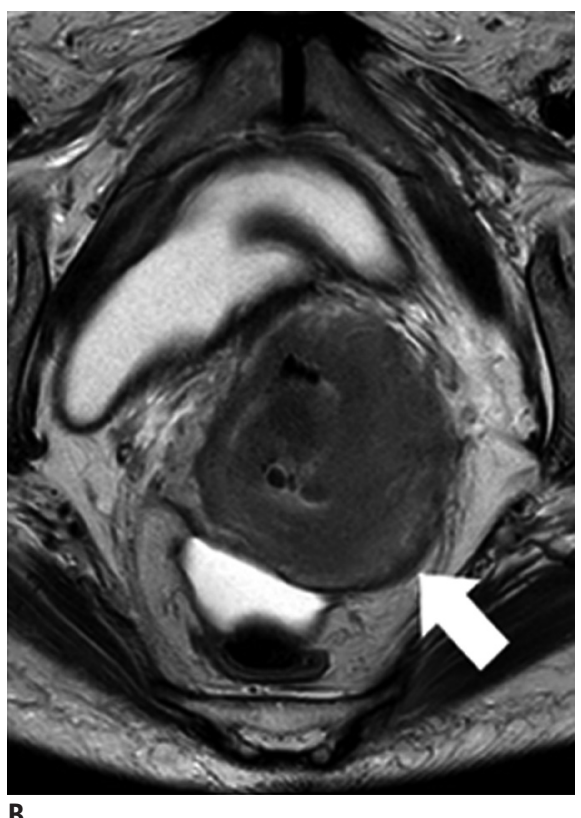

B
MRI findings widely vary from an expansile solid pattern and an invasive solid pattern to a diffuse infiltrative pattern (Fig. 1; Types 3-5). Generally, diffuse enhancement and low $A D C$ values are observed $(17,27)$.

Fig. 17. 63-year-old woman with primary lymphoma of uterus, diffuse large B-cell type.

Sagittal (A) and axial (B) T2WIs show infiltrating masses (Fig. 1; Type 5) located in cervix and corpus (arrows). C. Tumors show markedly restricted diffusion (arrow) on axial DWI, suggesting rich cellularity.

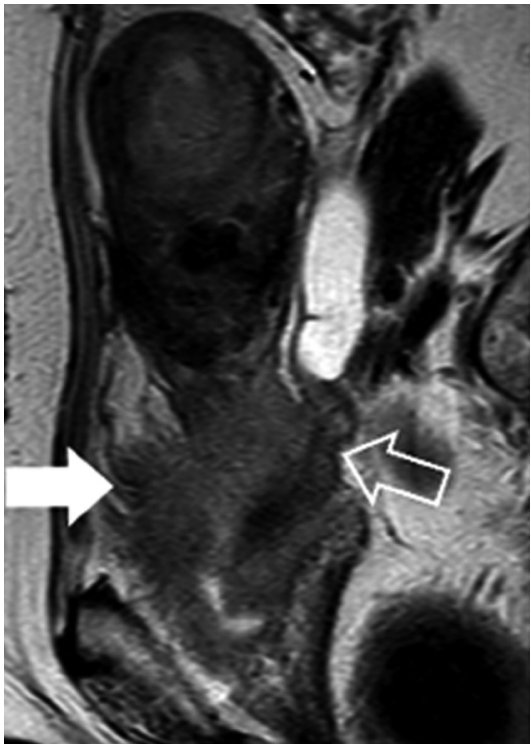

A

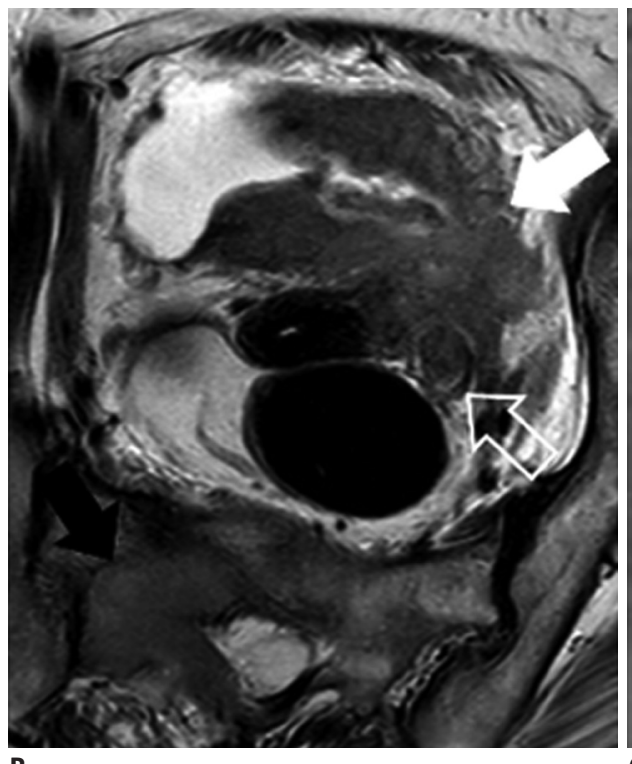

B

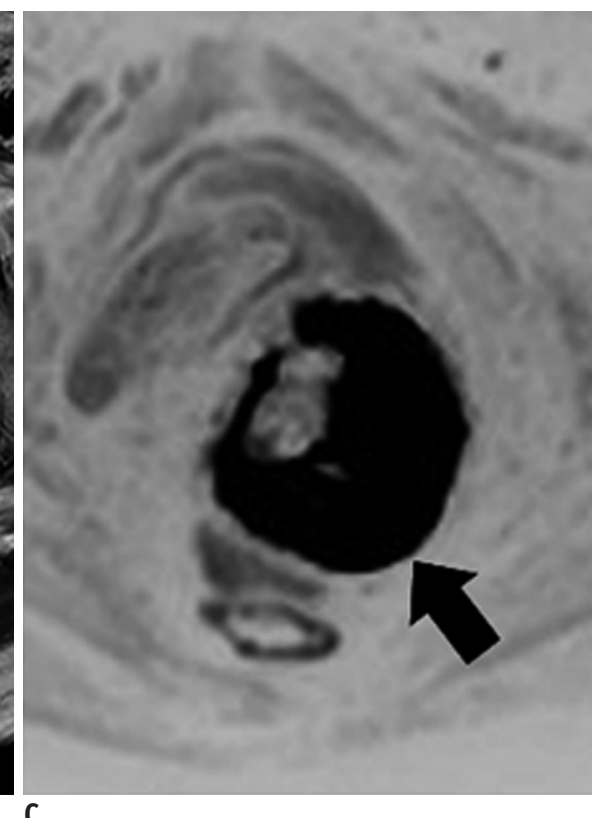

C 


\section{Secondary Tumors (Fig. 18)}

Malignancies arising from structures surrounding the cervix, such as the uterine body, bladder, ureter, rectum, and ovary, can be misdiagnosed as primary uterine cervical cancer because of uterine cervix involvement (28). MRI is a useful tool for distinguishing secondary cervical tumors from the primary ones.

In summary, uterine cervical adenocarcinoma has a variety of histopathological subtypes. Among them, GAS and VGC show relatively characteristic imaging findings, and their histological types can be suggested using MRI. GAS is not associated with high-risk HPV infection, and its incidence threatens to increase. It exhibits a poor prognosis and should be distinguished from LEGH, though this differentiation can be sometimes difficult by MRI alone. VGC, on the other hand, has an association with high-risk HPV infection and a good prognosis compared with SCC and the usual type adenocarcinoma. Various kinds of other tumors should be also differentiated on MRI, while it is currently difficult to distinguish them and HPV screening and pathological confirmation are usually necessary for definite diagnosis and further patient management.

\section{Conflicts of Interest}

The authors have no potential conflicts of interest to disclose.

\section{Acknowledgments}

We would like to thank Editage (www.editage.jp) for English language editing.

\section{ORCID iDs}

Tsukasa Saida

https://orcid.org/0000-0003-4530-7375

\section{REFERENCES}

1. Wilbur DC, Mikami Y, Colgan TJ, Park KJ, Ferenczy AS, Ronnett $\mathrm{BM}$, et al. Tumours of the uterine cervix: glandular tumours and precursors. In: Kurman RJ, Carcangiu ML, Herrington CS, Young RH, eds. WHO classification of tumours of female reproductive organs, 4th ed. Lyon: IARC Press, 2013:183-192

2. Smith HO, Tiffany MF, Qualls CR, Key CR. The rising incidence of adenocarcinoma relative to squamous cell carcinoma of the uterine cervix in the United States--a 24-year populationbased study. Gynecol Oncol 2000;78:97-105

3. Nogueira-Rodrigues A, Ferreira CG, Bergmann A, de Aguiar SS, Thuler LC. Comparison of adenocarcinoma (ACA) and squamous cell carcinoma (SCC) of the uterine cervix in a sub-optimally screened cohort: a population-based epidemiologic study of 51,842 women in Brazil. Gynecol Oncol 2014;135:292-296

4. Kong CS, McCluggage WG. Cervix. In: Mills SE, Greenson JK, Hornick JL, Longacre TA, Reuter VE, eds. Sternberg's diagnostic surgical pathology (Vol. 2), 6th ed. Philadelphia, PA: Wolters Kluwer Health, 2015:2419-2426

5. Stolnicu S, Barsan I, Hoang L, Patel P, Terinte C, Pesci A, et al. International Endocervical Adenocarcinoma Criteria and Classification (IECC): a new pathogenetic classification for invasive adenocarcinomas of the endocervix. Am J Surg Pathol 2018;42:214-226

6. Zhou J, Wu SG, Sun JY, Li FY, Lin HX, Chen QH, et al. Comparison of clinical outcomes of squamous cell carcinoma, adenocarcinoma, and adenosquamous carcinoma of the uterine cervix after definitive radiotherapy: a populationbased analysis. J Cancer Res Clin Oncol 2017;143:115-122

7. Noh JM, Park W, Kim YS, Kim JY, Kim HJ, Kim J, et al. Comparison of clinical outcomes of adenocarcinoma and adenosquamous carcinoma in uterine cervical cancer patients receiving surgical resection followed by radiotherapy: a multicenter retrospective study (KROG 13-10). Gynecol Oncol 2014;132:618-623

8. Epstein E, Di Legge A, Måsbäck A, Lindqvist PG, Kannisto P, Testa AC. Sonographic characteristics of squamous cell cancer and adenocarcinoma of the uterine cervix. Ultrasound Obstet Gynecol 2010;36:512-516

9. Kidd EA, Siegel BA, Dehdashti F, Grigsby PW. The standardized uptake value for $\mathrm{F}-18$ fluorodeoxyglucose is a sensitive predictive biomarker for cervical cancer treatment response and survival. Cancer 2007;110:1738-1744

10. Mitchell DG, Snyder B, Coakley F, Reinhold C, Thomas G, Amendola M, et al. Early invasive cervical cancer: tumor delineation by magnetic resonance imaging, computed tomography, and clinical examination, verified by pathologic results, in the ACRIN 6651/G0G 183 intergroup study. J Clin Oncol 2006;24:5687-5694

11. Kido A, Mikami Y, Koyama T, Kataoka M, Shitano F, Konishi I, et al. Magnetic resonance appearance of gastric-type adenocarcinoma of the uterine cervix in comparison with that of usual-type endocervical adenocarcinoma: a pitfall of newly described unusual subtype of endocervical adenocarcinoma. Int J Gynecol Cancer 2014;24:1474-1479

12. Shimada M, Kigawa J, Nishimura R, Yamaguchi S, Kuzuya K, Nakanishi T, et al. Ovarian metastasis in carcinoma of the uterine cervix. Gynecol Oncol 2006;101:234-237

13. Ando $H$, Miyamoto $T$, Kashima $H$, Takatsu A, Ishii K, Fujinaga $Y$, et al. Usefulness of a management protocol for patients with cervical multicystic lesions: a retrospective analysis of 94 cases and the significance of GNAS mutation. J Obstet Gynaecol Res 2016;42:1588-1598

14. Li H, Sugimura K, Okizuka H, Yoshida M, Maruyama R, Takahashi $\mathrm{K}$, et al. Markedly high signal intensity lesions in the uterine cervix on T2-weighted imaging: differentiation 
between mucin-producing carcinomas and nabothian cysts. Radiat Med 1999;17:137-143

15. Zhao L, Xu T, Cui M, Fu Z. A retrospective review of 11 cases of villoglandular papillary adenocarcinoma of the uterine cervix and a review of the literature. Oncol Lett 2016;11:21642168

16. Takeuchi M, Matsuzaki K, Bando Y, Sakaki M, Furumoto $H$, Harada M. Magnetic resonance manifestations of villoglandular papillary adenocarcinoma of the uterine cervix with a fernleaf-like appearance. Magn Reson Med Sci 2014;13:267-270

17. Okamoto Y, Tanaka Y0, Nishida M, Tsunoda H, Yoshikawa H, Itai Y. MR imaging of the uterine cervix: imaging-pathologic correlation. Radiographics 2003;23:425-445; quiz 534-535

18. Colgan TJ, Kim KR, Hirschowitz L, McCluggage WG. Tumours of the uterine cervix: other epithelial tumours. In: Kurman $\mathrm{RJ}$, Carcangiu ML, Herrington CS, Young RH, eds. WHO classification of tumours of female reproductive organs, 4th ed. Lyon: IARC Press, 2013:194

19. Colgan TJ, Kim I, Hirschowitz L, McCluggage WG. Tumours of the uterine cervix: neuroendocrine tumours. In: Kurman $\mathrm{RJ}$, Carcangiu ML, Herrington CS, Young RH, eds. WHO classification of tumours of female reproductive organs, 4th ed. Lyon: IARC Press, 2013:196-198

20. Lin Y, Lin WY, Liang JA, Lu YY, Wang HY, Tsai SC, et al. Opportunities for 2-[ $\left.{ }^{18} \mathrm{~F}\right]$ fluoro-2-deoxy-D-glucose PET/CT in cervical-vaginal neuroendocrine carcinoma: case series and literature review. Korean J Radiol 2012;13:760-770

21. Duan X, Ban X, Zhang X, Hu H, Li G, Wang D, et al. MR imaging features and staging of neuroendocrine carcinomas of the uterine cervix with pathological correlations. Eur Radiol
$2016 ; 26: 4293-4302$

22. Nucci MR, Nielsen GP, Carcangiu ML, Oliva E, Quade B. Tumours of the uterine cervix: mesenchymal tumours and tumour-like lesions. In: Kurman RJ, Carcangiu ML, Herrington CS, Young RH, eds. WHO classification of tumours of female reproductive organs, 4th ed. Lyon: IARC Press, 2013:198-199

23. Tanaka YO, Nishida M, Tsunoda H, Okamoto Y, Yoshikawa H. Smooth muscle tumors of uncertain malignant potential and leiomyosarcomas of the uterus: MR findings. J Magn Reson Imaging 2004;20:998-1007

24. Sato K, Yuasa N, Fujita M, Fukushima Y. Clinical application of diffusion-weighted imaging for preoperative differentiation between uterine leiomyoma and leiomyosarcoma. Am J Obstet Gynecol 2014;210:368.e1-e368.e8

25. Nucci MR, Ferry JA, Carcangiu ML, Oliva E, Quade B. Tumours of the uterine cervix: lymphoid and myeloid tumours. In: Kurman RJ, Carcangiu ML, Herrington CS, Young RH, eds. WHO classification of tumours of female reproductive organs, 4th ed. Lyon: IARC Press, 2013:205

26. Vang R, Medeiros LJ, Ha CS, Deavers M. Non-Hodgkin's lymphomas involving the uterus: a clinicopathologic analysis of 26 cases. Mod Pathol 2000;13:19-28

27. Marín C, Seoane JM, Sánchez M, Ruiz Y, García JA. Magnetic resonance imaging of primary lymphoma of the cervix. Eur Radiol 2002;12:1541-1545

28. Nucci MR, Ferry JA, Carcangiu ML, Oliva E, Quade B. Tumours of the uterine cervix: secondary tumours. In: Kurman RJ, Carcangiu ML, Herrington CS, Young RH, eds. WHO classification of tumours of female reproductive organs, 4th ed. Lyon: IARC Press, 2013:206 